\title{
Influence of vaccination status and clinical, seasonal and sociodemographic factors on rotavirus prevalence in KwaZulu-Natal, South Africa
}

\author{
Department of Medical Microbiology, University of KwaZulu-Natal, Durban, South Africa \\ *Corresponding author, Email: osemas2@gmail.com
}

Osaretin E Asowata*, Olubisi T Ashiru, Saajida Mahomed (D, A Willem Sturm (D) and Prashini Moodley

Check for updates

\begin{abstract}
Human rotavirus infection is the leading cause of diarrhoea in infants and young children worldwide. In South Africa, diarrhoea is a major cause of childhood morbidity and mortality in children less than five years old, and before the vaccine was introduced rotavirus had been reported as causing one-third of all diarrhoeal related hospital admissions. This study assessed factors influencing the prevalence of rotavirus in children aged five years and under in KwaZulu-Natal, South Africa between June 2014 and June 2015. In addition, genotypes of the rotaviruses were determined. A stool specimen was collected from children presenting with diarrhoea to a regional hospital. Clinical, vaccination status, seasonal and sociodemographic information was collected using a structured questionnaire. ELISA (enzyme linked immunosorbent assay) was performed to detect rotavirus antigen in the stool. Rotavirus from selected positives specimens were genotyped using RT-PCR (reverse transcriptase polymerase chain reaction). The data were analysed using SPSS. In total, 365 stool specimens were collected. Rotavirus antigen was detected in $83(23 \%)$ patients. The prevalence of rotavirus was not affected by vaccination status $(p=0.3 ;$ OR 1.5; Cl 0.7-3.1), HIV status ( $p=0.2 ; \mathrm{OR} 0.6 ; \mathrm{Cl} 0.2-1.5)$, breastfeeding ( $p=0.9 ; \mathrm{OR} 1.1 ; \mathrm{Cl} 0.5-2.5)$ and administration of anti-helminth treatment ( $p=0.6$; OR $0.8 ; \mathrm{Cl} 0.3-1.9)$. The highest rotavirus prevalence was observed in the winter season $(p<0.001$; OR 43.3; Cl 14.9-125.0). The G9P[8] was the most prevalent genotype (21\%) followed by G9P[4] (14\%). Rotavirus remains a major contributor to childhood diarrhoeal aetiology and hospitalisation in KwaZulu-Natal. Further investigation is needed to better understand the key drivers of rotavirus infection despite a successful vaccination programme in South Africa.
\end{abstract}

Key words children, KwaZulu-Natal, prevalence, rotavirus, sociodemographic factors, vaccination

\section{Introduction}

Rotavirus belongs to the family of Reoviridae. ${ }^{1}$ This family includes 12 genera, of which rotavirus is the most clinically significant and is the leading cause of acute infantile diarrhoea worldwide. ${ }^{1}$ Approximately 576,000 diarrhoeal deaths are recorded worldwide among children less than five years old annually and rotavirus accounts for 215,000 (37.3\%) of these deaths. ${ }^{2}$ About $50 \%$ of these rotavirus infections occur in developing countries. ${ }^{2}$ In South Africa, diarrhoeal diseases are ranked as the third cause of childhood mortality in children less than five years of age. ${ }^{3}$ Rotavirus has been documented as causing one-third of all admissions to hospital for diarrhoea prior to the introduction of the rotavirus vaccine. ${ }^{4}$

Two oral rotavirus vaccines have been licensed for global use. The monovalent Rotarix ${ }^{\circledast}$ (RV1) (GlaxoSmithKline Biologicals, Rixensart, Belguim) and the pentavalent RotaTeq ${ }^{\circledR}$ (RV5) (Merck and Co., Whitehouse Station, NJ, USA). The monovalent vaccine was introduced into the South African Expanded Programme for Immunization in 2009 and it is administered orally at 6 and 14 weeks of age. Both vaccines have been reported to have high efficacies in high-income countries such as the United States of America, Finland, Austria and Israel ${ }^{5,6}$ where the severity of rotavirus-associated diarrhoea is minimal. In contrast, reduced levels of vaccine protection have been recorded in low-income countries like Malawi, South Africa, Ghana, Kenya and Mali ${ }^{5,7,8}$ where rotavirus-associated diarrhoea is characterised by a higher burden of severe disease.

A great reduction in rotavirus-associated diarrhoeal hospitalisation was recorded in a study conducted among children less than five years old in three South African sentinel sites between 2009 and 2011 post-vaccination. ${ }^{9}$ However, an upsurge in rotavirus-associated diarrhoea cases in hospitals and clinics within the eThekwini metropolitan area in KwaZulu-Natal in $2013^{10}$ has raised significant questions regarding the impact of this vaccine, especially since a large proportion of the infected children had received two doses of the monovalent vaccine. Of the 242 stool samples tested as of July 3, 2013 at the National Institute of Communicable Diseases (NICD), rotavirus was detected in 55\% (134/242) of the samples with strains G2P[4] and G9P[8] detected in 54\% (72/ 134) and $39 \%(52 / 134)$ of cases respectively. ${ }^{10}$ Environmental enteropathy, a sub-clinical condition affecting the small intestine in children living in low-income countries, has been implicated as an important determinant of vaccine-induced protection. ${ }^{11}$ Great diversity has been observed in the intestinal microbial composition of children living in low-income countries when compared with the microbiota of children in high-income countries. $^{12}$ This may be the reason behind the observed differences in vaccine efficacy between the low- and middle-income countries and the high-income countries. However, the influence of the gut microbiome on the rotavirus vaccine efficacy is yet to be elucidated. Seroconversion of oral cholera vaccine was enhanced by treating with albendazole for intestinal helminth infestation before vaccine administration ${ }^{13}$ but it is unknown whether treatment with anti-helminth will have same effect on the rotavirus vaccine. Nutritional deficiencies of zinc, vitamin $A$ and vitamin $D$ have been reported to negatively affect the immune system. ${ }^{14}$ Consequently, this may also influence the rotavirus vaccine protection potential. ${ }^{14}$ Furthermore, maternal IgG and IgA, which is transferred through the placenta and via breast milk respectively, offer protection against rotavirus diarrhoea. However, these antibodies have been 
documented to compromise the immunogenicity of rotavirus vaccine in South Africa and Zambia. ${ }^{15-17}$

This study assessed the influence of vaccination status and clinical, seasonal and sociodemographic factors on rotavirus prevalence and determined the genotypes of these rotaviruses in children aged five years and under in KwaZulu-Natal.

\section{Methods}

\section{Ethics considerations}

This study was approved by the Biomedical Research Ethics Committee (BREC) of the University of KwaZulu-Natal (REF: BE222/13) in accordance with its ethical standards and with the 1964 Helsinki declaration and its later amendments or comparable ethical standards.

\section{Study site and patient population}

The study took place between June 2014 and June 2015 in the Paediatric Resuscitation Unit (PRU) in the outpatient department of King Edward VIII Hospital (KEH) in Durban, South Africa. KEH is a teaching hospital attached to the Nelson R Mandela School of Medicine at the University of KwaZulu-Natal (UKZN). The hospital has 922 beds and an annual outpatient attendance of about 360,000 patients. The hospital is situated in the eThekwini district of KwaZulu-Natal (KZN) province and provides regional and tertiary services to the whole of $K Z N$, as well as referrals from the neighbouring Eastern Cape province. The PRU is the first point of call for all cases and a decision is made by the paediatricians based on case severity whether a child should be observed for a few hours or admitted. Diarrhoea is diagnosed at the PRU and samples are collected accordingly. A total of $96 \%(351 / 365)$ of the children enrolled in this study were eventually admitted.

Written informed consent was obtained from parents or guardians of children aged five years and under presenting with diarrhoea from Monday till Friday from 8:00 till 14:00 between June 2014 and June 2015. Diarrhoea was defined by a paediatrician diagnosis, or by three or more loose stools within a 24-hour period. In some cases, these diarrhoeic children had accompanying symptoms of fever and/or vomiting. The body temperature was measured using an infrared ear thermometer (HuBDIC Co. $\mathrm{Ltd}$, Korea). Fever was defined as a temperature of $\geq 38.5^{\circ} \mathrm{C}{ }^{18}$ Clinical, vaccination status, seasonal and sociodemographic data were collected using a structured questionnaire. Some of these data, such as the patient's age, gender, HIV status, receipt of zinc supplements and anti-helminthic agents, vaccination status and mode of feeding were collected from the patient's hospital files and vaccination cards. HIV testing was done in the laboratory of $\mathrm{KEH}$ using blood specimens collected by means of the dry blood spot (DBS) collection kit. The DBS was used for HIV polymerase chain reaction (PCR) using the COBAS ${ }^{\varpi}$ AmpliPrep/COBAS ${ }^{\circledR}$ TaqMan $^{\circledR}$ HIV-1 Test, v2.0 (Roche, South Africa). Children who tested HIV-negative but had HIV-positive mothers were defined as HIV exposed uninfected. A child who tested HIV-negative and whose parents were HIV-negative was defined as HIV unexposed. Children who tested HIV-positive irrespective of the HIV status of their parents were defined as HIV infected. Children whose HIV status could not be ascertained were labelled as status unknown. Seasonality was defined according to the website of the South African Weather Services. ${ }^{19}$ Breastfeeding information were collected only from children less than six months old.

\section{Specimen collection}

Stool specimens were collected from soiled diapers of the children using a wooden spatula from Monday till Friday of the study period between 8:00 and 14:00 hours on day of admission. The stool was placed into sterile, leakproof containers. The specimens were transported to the Department of Medical Microbiology in UKZN in a cooler box with ice packs, which maintained a temperature below $8^{\circ} \mathrm{C}$. In the laboratory, the stool specimens were stored at $-80^{\circ} \mathrm{C}$ prior to analysis.

\section{Rotavirus detection and genotyping}

Suspensions containing approximately $10 \%$ stool in PBS (PH 7.2) were tested with the ProSpecT ${ }^{\mathrm{TM}}$ Rotavirus antigen detection kit (Oxoid Ltd, Basingstoke, UK). This enzyme-linked immunosorbent assay (ELISA) is recommended by the World Health Organization. $^{20}$ This assay utilises a polyclonal antibody to detect group-specific proteins, including the major inner capsid protein (VP6), present in Group A rotaviruses.

Viral ribonucleic acid (RNA) was extracted using the QIAamp viral mini-kit (Qiagen, Hilden, Germany). With this RNA as template, complementary deoxyribonucleic acid (cDNA) was synthesised using the high-capacity CDNA reverse transcription kit with RNase inhibitors (Thermo Fisher Scientific, Carlsbad, CA, USA). The cDNA from 21 ELISA-positive and 14 ELISA-negative specimens were used as template in a reverse transcriptase polymerase chain reaction (RT-PCR) to confirm the ELISA results. Primers for rotavirus VP7 (G-types) used are Beg 9, End 9, aBT1, aCT2, mG3, aDT4, aAT8v, mG9, mG10 and mG12b. ${ }^{20-24}$ The VP4 (P-types) primers used are Con3, Con2, 2T-1, 3T-1, 1T-1v, 4T-1, $5 \mathrm{~T}-1, \mathrm{mP}(11)$ and $\mathrm{P} 4943 .^{20,22,23,25-27}$ The genotyping was done using a conventional hemi-nested multiplex RT-PCR. The PCR products were subjected to electrophoresis in a $1.5 \%(\mathrm{w} / \mathrm{v})$ agarose gel in tris-acetate-EDTA (TAE) buffer with a $100 \mathrm{bp}$ DNA ladder (Sigma Aldrich, St Louis, MO, USA) as a molecular weight marker.

\section{Statistical analysis}

The sample size was calculated using the prevalence formulae by Hajian-Tilaki. ${ }^{28}$ We assumed a rotavirus prevalence of $25 \%$ and an ELISA sensitivity of $90 \%$. A sample size of 282 patients was needed to estimate the prevalence with $95 \%$ confidence. The association between the prevalence of rotavirus and the categorical variables was assessed using the Pearson chi-square test. A $p$-value $<0.05$ was considered to be statistically significant. Odds ratios and $95 \%$ confidence limits were used to measure the strength of the association. A multivariable logistic model was used to identify independent risk factors associated with rotavirus infection. All variables were included in the model with the exception of breastfeeding due to the missing values. The data were analysed using SPSS (version 22; IBM Corp, Armonk, NY, USA).

\section{Results}

In total 365 children aged 5 years and under were enrolled in this study. The majority of these children $(n=316,87 \%)$ were younger than 2 years. Rotavirus antigen was detected in the stool of $83(23 \%)$ children. Among the rotavirus- positive patients, $45(54 \%)$ were male and $38(46 \%)$ female $(p=0.5)$. The prevalence of rotavirus infection among the under two years and above two years age group was significantly different in the univariate but not in the multivariate analysis. The highest prevalence (30\%) was observed in children between 12 and 23 months old $(p=0.02)$ (Table 1). However, this observation was made only in the univariate analysis and not in the multivariate. 
Table 1: Clinical, vaccination status, seasonal and sociodemographic characteristics of study participants in KwaZulu-Natal

\begin{tabular}{|c|c|c|c|c|c|c|c|c|}
\hline \multirow{2}{*}{ Item } & \multirow[b]{2}{*}{ RV positive $(n=83)$} & \multirow[b]{2}{*}{ Total } & \multicolumn{3}{|c|}{ Univariate analysis } & \multicolumn{3}{|c|}{ Multivariate analysis } \\
\hline & & & OR & $95 \% \mathrm{Cl}$ & $\begin{array}{c}p- \\
\text { value }\end{array}$ & OR (adj) & $95 \% \mathrm{Cl}$ & $\begin{array}{c}p- \\
\text { value }\end{array}$ \\
\hline \multicolumn{9}{|l|}{ Gender: } \\
\hline Male & $45(22 \%)$ & 206 & 1 & & & 1 & & \\
\hline Female & $38(24 \%)$ & 159 & 1.1 & $(0.7-1.8)$ & 0.5 & 0.9 & $(0.5-1.8)$ & 0.8 \\
\hline \multicolumn{9}{|l|}{ Age groups (months): } \\
\hline$(0-11)$ & $49(22 \%)$ & 224 & 2.0 & $(0.8-5.0)$ & 0.13 & 1.9 & $(0.6-6.0)$ & 0.3 \\
\hline$(12-23)$ & $28(30 \%)$ & 92 & 3.1 & $(1.2-8.2)$ & 0.02 & 2.3 & $(0.7-6.9)$ & 0.1 \\
\hline$(24-60)$ & $6(12 \%)$ & 49 & 1 & & & 1.0 & & \\
\hline \multicolumn{9}{|l|}{ Vaccination status: } \\
\hline Completely/incompletely vaccinated & $64(25 \%)$ & 260 & 1.5 & $(0.8-2.6)$ & 0.2 & 1.5 & $(0.7-3.1)$ & 0.3 \\
\hline Not vaccinated & $19(18 \%)$ & 105 & 1.0 & & & 1.0 & & \\
\hline \multicolumn{9}{|l|}{ HIV infection status: } \\
\hline HIV infected & $9(23 \%)$ & 39 & 0.9 & $(0.4-2.1)$ & 0.8 & 0.6 & $(0.2-1.5)$ & 0.2 \\
\hline HIV unexposed & $50(25 \%)$ & 203 & 1 & & & 1.0 & & \\
\hline HIV exposed uninfected & $13(24 \%)$ & 55 & 0.9 & $(0.5-1.9)$ & 0.9 & 0.7 & $(0.3-1.6)$ & 0.4 \\
\hline Status unknown & $11(16 \%)$ & 68 & 0.6 & $(0.3-1.2)$ & 0.2 & 1.1 & $(0.4-2.7)$ & 0.9 \\
\hline \multicolumn{9}{|l|}{ Zinc supplementation: } \\
\hline Zinc given & $51(28 \%)$ & 183 & 2.4 & $(1.3-4.2)$ & 0.003 & 2.1 & $(1.0-4.1)$ & 0.04 \\
\hline Zinc not given & $20(14 \%)$ & 142 & 1 & & & 1.0 & & \\
\hline Status unknown & $12(30 \%)$ & 40 & 2.6 & $(1.1-6.0)$ & 0.02 & 1.1 & $(0.4-3.0)$ & 0.8 \\
\hline \multicolumn{9}{|l|}{ Anti-helminth treatment: } \\
\hline Anti-helminth given & $16(23 \%)$ & 69 & 1.1 & $(0.6-2.0)$ & 0.8 & 0.8 & $(0.3-1.9)$ & 0.6 \\
\hline Anti-helminth not given & $61(22 \%)$ & 277 & 1 & & & 1 & & \\
\hline Status unknown & $6(32 \%)$ & 19 & 1.6 & $(0.6-4.5)$ & 0.3 & 2.2 & $(0.5-9.1)$ & 0.3 \\
\hline \multicolumn{9}{|l|}{ Seasonality: } \\
\hline Winter & 79 (46\%) & 172 & 40.1 & $(14.3-113.0)$ & $<0.001$ & 43.3 & $(14.9-125.0)$ & $<0.001$ \\
\hline Spring/summer/autumn & $4(2 \%)$ & 193 & 1 & & & 1 & & \\
\hline
\end{tabular}

RV: rotavirus; OR: odds ratio; OR (adj): adjusted odds ratio; Cl: confidence interval; $p$-value: measure of significance $(p<0.05)$.

A total of 260 patients $(71 \%)$ received at least one dose of rotavirus vaccine and among these, $64 / 260$ (25\%) were positive for rotavirus antigen. The prevalence of rotavirus infection did not differ between children who were vaccinated and children who were not vaccinated in either the univariate $(p=0.2)$ or

Table 2: Genotypes of rotaviruses in KwaZulu-Natal $(n=29)$

\begin{tabular}{|l|c|}
\hline G/P-types & $\mathbf{n}(\%)$ \\
\hline G9P[8] & $6(20.7)$ \\
\hline G9P[4] & $4(13.8)$ \\
\hline G12P[4] & $3(10.3)$ \\
\hline G9P[6] & $2(6.9)$ \\
\hline G4/8P[6] & $1(3.4)$ \\
\hline G4P[6] & $1(3.4)$ \\
\hline G12P[6] & $1(3.4)$ \\
\hline G8P[10] & $1(3.4)$ \\
\hline G9P[10] & $1(3.4)$ \\
\hline G9P[NT] & $4(13.8)$ \\
\hline G12P[NT] & $1(3.4)$ \\
\hline G8[NT] & $1(3.4)$ \\
\hline G1P[NT] & $1(3.4)$ \\
\hline NTP[4] & $2(6.9)$ \\
\hline T:
\end{tabular}

NT: non-typeable, n: total number of genotypes. multivariate $(p=0.3)$ analyses. Of the total number of children enrolled in the study, $166(46 \%)$ were $\leq 6$ months of age and their mode of feeding was ascertained. There was no difference in the prevalence of rotavirus infection between children who were breastfed (17/68 [25\%]) and those who were not (26/98 [27\%]) $(p=0.9)$. The HIV status of the children had no influence on the rotavirus prevalence in the univariate $(p=0.8)$ and multivariate $(p=0.2)$ analyses between the HIV-infected and unexposed children. There was a higher rotavirus prevalence among children who had received zinc supplementation in comparison with those who did not in the univariate $(p=0.003)$ and multivariate model $(p=0.04)$. The prevalence of rotavirus infection among children who had been previously treated for possible helminth infestation did not differ from those who were not in both the univariate $(p=0.8)$ and multivariate $(p=0.6)$ analyses. Rotavirus-associated diarrhoea had a higher prevalence in winter in comparison with the other seasons $(p<0.001)$ in the univariate and multivariate analyses.

Of the 35 specimens tested by RT-PCR (21 ELISA-positive and 14 ELISA-negative), 29 (83\%) were positive. All ELISA-positive samples were PCR positive. However, eight ELISA-negative samples were PCR positive. Among these 29, 5 G types and 4 P-types were observed with G9P[8] as the dominant genotype. From one of the viruses the G-type could not be established with the available primers. Similarly, four did not reveal a Ptype (Table 2). 


\section{Discussion}

In low-income countries, the level of protection conferred by the monovalent rotavirus vaccine is lower ${ }^{29}$ as compared with that in high-income countries. ${ }^{30,31}$ This was highlighted in our study where $25 \%$ of children who received at least one dose of the Rotarix ${ }^{\oplus}$ vaccine had rotavirus-associated diarrhoea. The reason for this is still obscure. This lower protection is not unique to the rotavirus vaccine since it has also been reported for oral polio and cholera vaccines. ${ }^{32}$ A multitude of factors have been documented as likely causes for this low vaccine effectiveness. Some of these factors may be influenced by socioeconomic circumstances, including environmental and host factors, and have been suggested as possible determinants of protection conferred by oral vaccines. ${ }^{14}$ Despite these shortcomings, the rotavirus vaccine does still have significant impact on prevention of hospitalisation and mortality in these lowincome countries such as those in sub-Saharan Africa. ${ }^{33-37}$ In the children with diarrhoea reported in 2013 in KZN, 55\% of cases were caused by rotavirus ${ }^{10}$ and most of these were of the G2P[4] $(54 \% ; 72 / 134)$ and G9P[8] $(39 \% ; 52 / 134) .{ }^{10}$ This is despite the introduction of rotavirus vaccination four years earlier. Although the vaccination status of the children in this possible outbreak was not reported, they speculated that the increasing rotavirus cases could be due to an accumulation of unimmunised susceptible children, partial vaccine protection and variation in the severity of the rotavirus season. ${ }^{10}$

In this study, the influence of vaccination status and clinical, seasonal and sociodemographic factors on rotavirus prevalence was evaluated in a group of patients in KZN. With a $23 \%$ prevalence, rotavirus is still a major cause of diarrhoea among infants and young children in South Africa. A similar prevalence was reported from Malawi. ${ }^{38}$ In our study population, rotavirus infection was not associated with gender or HIV status. Similarly, a study conducted in Tanzania found that HIV infection did not increase the prevalence of rotavirus infection in patients with diarrhoea. However, the same study reported a higher prevalence among HIV-infected children without diarrhoea than in HIV-uninfected asymptomatic children. ${ }^{39}$

Prior to the introduction of rotavirus vaccination, all children in South Africa became infected with rotavirus before their third year of life. ${ }^{40}$ In low-income countries children acquire rotavirus infection at an earlier age than those in high-income countries. In this study, a significantly higher prevalence of rotavirus among children aged 12-23 months was observed in the univariate analysis but this was not significant in the multivariate model. We also found an increasing rotavirus prevalence from birth until the end of the second year of life. Above this age, the prevalence declined. The higher prevalence after the first year of life may be attributed to waning immunity elicited by natural infection or vaccination. The last dose of the Rotarix ${ }^{\circledR}$ vaccine is administered at 14 weeks of age in South Africa and in a few other countries. ${ }^{41,42} \mathrm{~A}$ booster dose has been suggested for older children in South Asia and some other developing countries before the rotavirus season commences. ${ }^{43,44}$ In most parts of South Africa, ${ }^{45}$ the prevalence of rotavirus-associated diarrhoea in KZN peaks in the cold season. This finding is useful when making recommendations to the Department of Health regarding the appropriate timing of booster doses in children above two years of age.

Nutritional deficiency is one of the factors that has been proposed to affect the protection induced by the rotavirus vaccine in low-income settings. Noteworthy is zinc deficiency, which has been reported to affect the immune system. ${ }^{14}$ We found that children who received zinc supplementation were more likely to be infected with rotavirus when compared with those who did not. This finding is discordant with previous findings, ${ }^{14}$ where a higher prevalence of rotavirus was detected among children who did not receive zinc supplementation. However, in a large proportion of the rotavirus-infected children $(14 \%)$ it was unknown whether zinc supplementation had been received. Nonetheless, the effect of zinc and other micronutrients on rotavirus prevalence and oral vaccine immunogenicity needs further exploration.

Intestinal colonisation with microorganisms following birth has a significant impact on intestinal immunity. In low-income countries, intestinal colonisation is driven by environmental enteropathy. This condition is characterised by chronic exposure to faecal pathogens due to poor sanitation, leading to chronic inflammation of the intestine. ${ }^{46,47}$ It is believed that Gram-negative bacterial components such as lipopolysaccharides mediate this inflammation, resulting in an increase in the release of pro-inflammatory cytokines and inflammatory cell infiltration of the gut, subsequently leading to a T-cell-mediated enteropathy. ${ }^{11}$ This condition has been studied extensively in Gambian infants. ${ }^{48}$ Although the immunological effects of environmental enteropathy and its relationship with poor nutrition and poor growth is well described, its role in reducing rotavirus and other oral vaccines effectiveness is not fully understood. Bacteria and helminth colonisation as early as 4 weeks of life have been found to significantly alter the numbers of memory B-cells when the infant reaches 18 months of age. ${ }^{49}$ The difference in intestinal organisms between populations of low- and high-income countries may directly influence the difference in rotavirus vaccine efficacy in these settings as recently reported..$^{45}$ Consequently, a lower vaccine efficacy may lead to continuous circulation of rotavirus strains in low-income countries. Treatment for intestinal helminths with albendazole before the administration of oral cholera vaccine has been shown to enhance the rate of seroconversion by this vaccine. ${ }^{13}$ Therefore, rotavirus-associated diarrhoea cases were stratified based on receipt of anti-helminthic treatment before admission. We did not find a relationship between the two. In addition, we could not ascertain whether an anti-helminthic drug was given before vaccination or afterwards. However, we found only a $1 \%$ prevalence of Ascaris lumbricoides in our study population (data not shown). It will be important to conduct further studies on the effect of intestinal bacteria and parasites on rotavirus vaccination.

Maternal antibodies acquired transplacentally and via breastfeeding have been documented to protect infants and young children from diseases. ${ }^{50}$ In this study, the mode of feeding of children who were six months old and younger was recorded. We found that exclusive breastfeeding or mixed feeding had no effect on rotavirus prevalence when compared with the rotavirus prevalence in formula-fed children. However, it has been reported that the immunogenicity of rotavirus vaccine may be compromised by maternal antibodies from breast milk, thus predisposing children to rotavirus infection. ${ }^{50}$

The Rotarix ${ }^{\circledast}$ vaccine schedule in South Africa is 6 weeks of age for the first dose and 14 weeks of age for the second dose. This differs from the schedule that was used in the efficacy trial of this vaccine in Africa, which was 10 and 14 weeks of age for the first and second dose respectively. ${ }^{5}$ The degree of protection offered by the 6 and 14 weeks schedule was only established in the post-vaccination effectiveness study in South Africa. ${ }^{29}$ 
Noteworthy is the fact that the immunogenicity of the Rotarix ${ }^{\circledast}$ vaccine when co-administered with OPV in completely vaccinated children at 6 and 10 weeks of age was lower than that of children vaccinated at 10 and 14 weeks of age with a $36 \%$ and $61 \%$ seroconversion rate respectively. ${ }^{51}$ When our study population was grouped based on vaccination status, it was found that this vaccine had no significant effect on the prevalence of rotavirus-associated diarrhoea. KZN has been reported to have a high (97.8\%) immunisation coverage ${ }^{52}$ and it is therefore difficult to imagine that a failure in the immunisation programme could explain our findings. In contrast, our study showed a vaccination coverage of only $71 \%$. Consequently, proper monitoring and reporting of vaccination coverage in South Africa need to be prioritised. In addition, the different vaccination schedules need to be further evaluated to maximise the potential benefit that this vaccine may offer.

The high cost of the global rotavirus vaccines has led to the production of more affordable country-specific vaccines. Examples of these rotavirus vaccines are the Lanzhou lamb rotavirus (LLR) vaccine, which has been in use in China since 2000, ${ }^{53}$ Rotavac vaccine, which was licensed by the Drugs Controller General of India in early 2014 and was introduced into its national immunisation programme in $2016^{54,55}$ and the RV3BB human neonatal rotavirus vaccine, which was found to be highly effective based on a clinical trial conducted in Indonesia. ${ }^{56}$ These vaccines were designed using prevalent strains in these countries. However, the year to year, season to season and region to region variations in the circulating genotypes of rotaviruses in low-income countries are possible factors influencing vaccine protection in these settings. The characteristic diversity of circulating rotavirus genotypes within confined geographical locations was highlighted in this study. We found $5 \mathrm{G}-$ types, 4 P-types and some non-typeable strains within the small group of 29 rotavirus-positive samples that were genotyped. The most prevalent genotype in our setting was G9P[8] (20.7\%) which is similar to that recorded in $2013,{ }^{10}$ where it was reported as the second most prevalent genotype after G2P[4]. However, this correlated with previous findings in other Southern African countries, namely Zimbabwe and Zambia, where G9P [8] accounted for $20 \%$ of the circulating genotypes. ${ }^{57}$ The genotype G1P[8] remains the most prevalent genotype worldwide. ${ }^{58-}$ ${ }^{60}$ A previous study reported that rotavirus strain diversity has no effect on the effectiveness of the RV1 and RV5 in high- and middle-income countries, ${ }^{61}$ thus excluding vaccine-induced selective pressure as a possible reason for the rotavirus strain diversity in low-income countries compared with the highincome countries.

A major limitation of this study is that it was conducted in one region of South Africa and may not be generalisable to the whole of South Africa. Our study may have been inadequately powered for some of the analyses. In addition, our inability to genotype all the positive rotaviruses detected has limited the provision of a true description of the circulating genotypes in KZN.

\section{Conclusion}

Rotavirus-associated diarrhoea is still a major contributor to childhood hospitalisation in KwaZulu-Natal, South Africa. Seasonality remain a key driver of rotavirus prevalence even in this post-vaccination era. A monovalent/polyvalent oral vaccine combined with a parenterally administered vaccine in a prime-boost schedule could be considered to improve rotavirus vaccine efficacy. Continuous surveillance of circulating strains is crucial to monitor the long-term impact of the rotavirus vaccines and identify any strains that escape protection, should they occur. The effect of a booster dose preceding each cold season in children up to the age of two years could also be investigated. Interventions targeted at providing oral rehydration solution (ORS), improving nutrition and improving socioeconomic conditions in low- and middle-income countries may be a starting point towards the prevention of rotavirus infection in countries where RV vaccination is yet to be introduced in the expanded programme on immunisation (EPI), and improved $\mathrm{RV}$ vaccine effectiveness in countries where this vaccine is already part of the EPI.

\section{Meetings where part of the information has been presented}

- Translational Vaccinology for Global Health (S1) - Keystone Symposia, Park Plaza Riverbank London, UK. Poster presentation (October 25-29, 2016).

- 6th Federation of Infectious Disease Society of Southern Africa (FIDSSA) congress, Drakensberg, KwaZulu-Natal. Poster presentation (November 5-8, 2015).

- 12th International Double-Stranded RNA (dsRNA) Virus Symposium in Goa, India. Poster presentation (October 6-10, 2015).

Acknowledgement - The authors wish to acknowledge the assistance provided by Dr Moherndran Archary, Dr Shashikant Ramji and all of the staff of the Paediatric outpatient Department of King Edward VIII Hospital, University of KwaZulu-Natal. In addition, the authors wish to acknowledge the assistance of Ms Cathy Connolly of the Department of Public Health Medicine in the University of KwaZulu-Natal, South Africa.

Disclosure statement - No potential conflict of interest was reported by the authors.

Funding - This research did not receive any specific grant from funding agencies in the public, commercial, or not-for-profit sectors.

\section{ORCID}

Saajida Mahomed (1D http://orcid.org/0000-0001-7095-9186 A Willem Sturm (1D) http://orcid.org/0000-0001-7521-8248

\section{References}

1. Estes MK, Kapikian A. Rotaviruses. In: Knipe DM, Howley PM, Griffin $\mathrm{DE}$, Lamb RA, Martin MA, Roizman B, editors. Fields virology. 5th ed. Philadelphia, Pennsylvania, USA: Wolters Kluwer Health/Lippincott Williams and Wilkins; 2007. p. 1917-75.

2. Tate JE, Burton $\mathrm{AH}$, Boschi-Pinto $\mathrm{C}$, et al. Global, regional, and national estimates of rotavirus mortality in children $<5$ years of age, 2000 2013. Clin Infect Dis. 2016;62(suppl 2):S96-S105. doi:10.1093/cid/ civ1013

3. UNICEF South Africa - Child and maternal health - Overview: Child and maternal health. [cited 22 Jan 2018]. https://www.unicef.org/ southafrica/survival_devlop_343.html

4. Steele AD, Peenze I, de Beer MC, et al. Anticipating rotavirus vaccines: epidemiology and surveillance of rotavirus in South Africa. Vaccine. 2003;21(5-6):354-60. http://www.ncbi.nlm.nih.gov/ pubmed/12531632

5. Madhi SA, Cunliffe NA, Steele D, et al. Effect of human rotavirus vaccine on severe diarrhea in African infants. $N$ Engl J Med. 2010;362(4):289-98. doi:10.1056/NEJMoa0904797

6. Vesikari T, Itzler R, Matson DO, et al. Efficacy of a pentavalent rotavirus vaccine in reducing rotavirus-associated health care utilization across 
three regions (11 countries). Int J Infect Dis. 2007;11(SUPPL 2). doi:10. 1016/S1201-9712(07)60019-8

7. Sow SO, Tapia M, Haidara FC, et al. Efficacy of the oral pentavalent rotavirus vaccine in Mali. Vaccine. 2012;30:A71-8. doi:10.1016/J. VACCINE.2011.11.094

8. Armah GE, Sow SO, Breiman RF, et al. Efficacy of pentavalent rotavirus vaccine against severe rotavirus gastroenteritis in infants in developing countries in sub-Saharan Africa: a randomised, double-blind, placebo-controlled trial. Lancet. 2010;376(9741):606-14. doi:10. 1016/S0140-6736(10)60889-6

9. Msimang VMY, Page N, Groome MJ, et al. Impact of rotavirus vaccine on childhood diarrheal hospitalization after introduction into the South African public immunization program. Pediatr Infect Dis J. 2013;32(12):1359-64. doi:10.1097/INF.0b013e3182a72fc0

10. National Institute of Communicable Diseases. Communicable diseases Communiqué. Commun Dis Commun. 2013;12(7):1-2. http:// www.nicd.ac.za/assets/files/NICD-NHLS\%25\%0A20Communicable\% 2520Disease\%2520Communiqu\%25\%0AC3\%25A9_June\% 25202013_\%25281\%2529.pdf\%0A)

11. Campbell DI, Murch SH, Elia M, et al. Chronic T cell-mediated enteropathy in rural west African children: relationship with nutritional status and small bowel function. Pediatr Res. 2003;54(3):306-11. doi:10.1203/01.PDR.0000076666.16021.5E

12. Lin A, Bik EM, Costello EK, et al. Distinct distal gut microbiome diversity and composition in Healthy children from Bangladesh and the United States. PLoS One. 2013;8(1):e53838. doi:10.1371/journal. pone. 0053838

13. Cooper PJ, Chico ME, Losonsky G, et al. Albendazole treatment of children with Ascariasis enhances the vibriocidal antibody response to the live attenuated oral cholera vaccine CVD 103-HgR. J Infect Dis. 2000;182(4):1199-206. doi:10.1086/315837

14. Clarke $E$, Desselberger U. Correlates of protection against human rotavirus disease and the factors influencing protection in lowincome settings. Mucosal Immunol. 2015;8(1):1-17. doi:10.1038/mi. 2014.114

15. Moon S-S, Groome MJ, Velasquez DE, et al. Prevaccination rotavirus serum $\lg G$ and $\lg A$ are associated with lower immunogenicity of live, oral human rotavirus vaccine in South African infants. Clin Infect Dis. 2016;62(2):157-65. doi:10.1093/cid/civ828

16. Chilengi $R$, Simuyandi $M$, Beach $L$, et al. Association of maternal immunity with rotavirus vaccine immunogenicity in Zambian infants. PLoS One. 2016;11(3):e0150100. doi:10.1371/journal.pone. 0150100

17. Mwila-Kazimbaya K, Garcia MP, Bosomprah S, et al. Effect of innate antiviral glycoproteins in breast milk on seroconversion to rotavirus vaccine (Rotarix) in children in Lusaka, Zambia. PLoS One. 2017;12 (12):e0189351. doi:10.1371/journal.pone.0189351

18. Binz $P$, Bodmer N, Leibundgut $K$, et al. Different fever definitions and the rate of fever and neutropenia diagnosed in children with cancer: a retrospective two-center cohort study. Pediatr Blood Cancer. 2013;60(5):799-805. doi:10.1002/pbc.24380

19. South African Weather Service. Publications. [cited 23 May 2018]. http://www.weathersa.co.za/climate/publications

20. WHO. Manual of rotavirus detection and characterization methods. Biologicals. 2009. p. 2-90

21. Iturriza-Gómara M, Kang G, Gray J. Rotavirus genotyping: keeping up with an evolving population of human rotaviruses. J Clin Virol. 2004;31(4):259-65. doi:10.1016/j.jcv.2004.04.009

22. Padilla-Noriega L, Werner-Eckert R, Mackow ER, et al. Serologic analysis of human rotavirus serotypes $\mathrm{P} 1 \mathrm{~A}$ and $\mathrm{P} 2$ by using monoclonal antibodies. J Clin Microbiol. 1993;31(3):622-8. http://www.ncbi.nlm. nih.gov/pubmed/7681440

23. Greenberg H, McAuliffe V, Valdesuso J, et al. Serological analysis of the subgroup protein of rotavirus, using monoclonal antibodies. Infect Immun. 1983;39(1):91-9. http://www.ncbi.nlm.nih.gov/pubmed/ 6185436

24. Santos N, Hoshino Y. Global distribution of rotavirus serotypes/genotypes and its implication for the development and implementation of an effective rotavirus vaccine. Rev Med Virol. 2005;15(1):29-56. doi:10. 1002/rmv.448

25. Iturriza-Gómara M, Green J, Brown DW, et al. Diversity within the VP4 gene of rotavirus $P[8]$ strains: implications for reverse transcription-
PCR genotyping. J Clin Microbiol. 2000;38(2):898-901. http://www. ncbi.nlm.nih.gov/pubmed/10655412

26. Gouvea V, Glass Rl, Woods $\mathrm{P}$, et al. Polymerase chain reaction amplification and typing of rotavirus nucleic acid from stool specimens. $J$ Clin Microbiol. 1990;28(2):276-82. http://www.ncbi.nlm.nih.gov/ pubmed/2155916

27. Gentsch JR, Woods PA, Ramachandran $M$, et al. Review of $G$ and $P$ typing results from a global collection of rotavirus strains: implications for vaccine development. J Infect Dis. 1996;174(Suppl 1): S30-6. http://www.ncbi.nlm.nih.gov/pubmed/8752288

28. Hajian-Tilaki K. Sample size estimation in diagnostic test studies of biomedical informatics. J Biomed Inform. 2014;48:193-204. doi:10. 1016/j.jbi.2014.02.013

29. Groome MJ, Page N, Cortese MM, et al. Effectiveness of monovalent human rotavirus vaccine against admission to hospital for acute rotavirus diarrhoea in South African children: a case-control study. Lancet Infect Dis. 2014;14(11):1096-104. doi:10.1016/S1473-3099(14)70940-5

30. Staat MA, Payne DC, Donauer S, et al. Effectiveness of pentavalent rotavirus vaccine against severe disease. Pediatrics. 2011;128(2): e267-75. doi:10.1542/peds.2010-3722

31. Buttery JP, Lambert SB, Grimwood K, et al. Reduction in rotavirus-associated acute gastroenteritis following introduction of rotavirus vaccine into Australia's national childhood vaccine schedule. Pediatr Infect Dis J. 2011;30(1 Suppl):S25-9. doi:10.1097/INF.0b013e3181fefdee

32. Serazin AC, Shackelton LA, Wilson C, et al. Improving the performance of enteric vaccines in the developing world. Nat Immunol. 2010;11(9):769-73. doi:10.1038/ni0910-769

33. Tsolenyanu E, Djadou KE, Fiawoo M, et al. Evidence of the impact of monovalent rotavirus vaccine on childhood acute gastroenteritis hospitalization in Togo. Vaccine. February 2018. doi:10.1016/J. VACCINE.2018.01.058

34. Enweronu-Laryea CC, Armah G, Sagoe KW, et al. Sustained impact of rotavirus vaccine introduction on rotavirus gastroenteritis hospitalizations in children. Vaccine. May 2018. doi:10.1016/J.VACCINE.2018.02. 058

35. Lyamuya F, Michael F, Jani B, et al. Trends in diarrhea hospitalizations among infants at three hospitals in Tanzania before and after rotavirus vaccine introduction. Vaccine. April 2018. doi:10.1016/J. VACCINE.2017.11.051

36. Mukaratirwa A, Berejena C, Nziramasanga $P$, et al. Distribution of rotavirus genotypes associated with acute diarrhoea in Zimbabwean children less than five years old before and after rotavirus vaccine introduction. Vaccine. April 2018. doi:10.1016/J.VACCINE.2018.03.069

37. Wandera EA, Mohammad S, Bundi $M$, et al. Impact of rotavirus vaccination on rotavirus hospitalisation rates among a resource-limited rural population in Mbita, Western Kenya. Trop Med Int Heal. 2018;23(4):425-32. doi:10.1111/tmi.13040

38. Bar-Zeev N, Kapanda L, Tate JE, et al. Effectiveness of a monovalent rotavirus vaccine in infants in Malawi after programmatic roll-out: an observational and case-control study. Lancet Infect Dis. 2015;15 (4):422-8. doi:10.1016/S1473-3099(14)71060-6

39. Moyo SJ, Blomberg B, Hanevik K, et al. Genetic diversity of circulating rotavirus strains in Tanzania prior to the introduction of vaccination. PLoS One. 2014;9(5):e97562. doi:10.1371/journal.pone.0097562

40. Seheri LM, Page NA, Mawela MPB, et al. Rotavirus vaccination within the South African expanded programme on immunisation. Vaccine. 2012;30:C14-C20. doi:10.1016/J.VACCINE.2012.04.018

41. WHO vaccine-preventable diseases: monitoring system. 2015 globa summary. Immunization schedules by antigens. 2015. http://apps. who.int/immunization_monitoring/globalsummary/schedules?sc\% 255Bc\%255D\%255B\%255D=SDN\&sc\%255Bd\%255D=\&sc\%255Bv\% 255D\%255B\%255D=BCG\&sC\%255Bv\%255D\%255B\%255D= CHOLERA\&SC\%255Bv\%255D\%255B\%255D=DIP\&sC\%255Bv\%255D\% 255B\%255D=DT\&sc\%255Bv\%255D\%255B\%255D=DTAP\&.

42. Mwenda JM, Burke RM, Shaba K, et al. Implementation of rotavirus surveillance and vaccine introduction - World Health Organization African region, 2007-2016. MMWR Morb Mortal Wkly Rep. 2017;66(43):1192-6. doi:10.15585/mmwr.mm6643a7

43. Jagai JS, Sarkar R, Castronovo D, et al. Seasonality of rotavirus in South Asia: a meta-analysis approach assessing associations with temperature, precipitation, and vegetation index. PLoS One. 2012;7 (5):e38168. doi:10.1371/journal.pone.0038168 
44. Burnett E, Lopman BA, Parashar UD. Potential for a booster dose of rotavirus vaccine to further reduce diarrhea mortality. Vaccine. 2017;35(51):7198-203. doi:10.1016/J.VACCINE.2017.10.027

45. Page N, Mapuroma F, Seheri M, et al. Rotavirus surveillance report, South Africa, 2013. Commun Dis Surveill Bull. 2013;12(4):108-13.

46. Neto UF, Martins MC, Lima FL, et al. Asymptomatic environmental enteropathy among slum-dwelling infants. J Am Coll Nutr. 1994;13 (1):51-6. doi:10.1080/07315724.1994.10718371

47. Harris V, Ali A, Fuentes S, et al. Rotavirus vaccine response correlates with the infant gut microbiota composition in Pakistan. Gut Microbes. September 2017:1-9. doi:10.1080/19490976.2017.1376162

48. Lunn PG, Northrop-Clewes CA. Intestinal permeability: update on the enzymatic assay of mannitol. Clin Chim Acta. 1992;205(1-2):151-2. [cited 2018 Jan 22]. http://www.ncbi.nlm.nih.gov/pubmed/1521336

49. Lundell A-C, Björnsson V, Ljung A, et al. Infant B cell memory differentiation and early gut bacterial colonization. J Immunol. 2012;188 (9):4315-22. doi:10.4049/jimmunol.1103223

50. Moon S-S, Wang Y, Shane AL, et al. Inhibitory effect of breast milk on infectivity of live oral rotavirus vaccines. Pediatr Infect Dis J. 2010;29 (10):919-23. doi:10.1097/INF.0b013e3181e232ea

51. Steele AD, De Vos B, Tumbo J, et al. Co-administration study in South African infants of a live-attenuated oral human rotavirus vaccine (RIX4414) and poliovirus vaccines. Vaccine. 2010;28(39):6542-8. doi:10.1016/J.VACCINE.2008.08.034

52. van den Heever J. What is the South African Immunisation coverage? 2012;(October). https://africacheck.org/wp-content/uploads/2012/ 11/S-Africans-Immunization-Coverage-ppt-EPI-Manager3.pdf

53. Fu C, He Q, Xu J, et al. Effectiveness of the Lanzhou lamb rotavirus vaccine against gastroenteritis among children. Vaccine. 2012;31 (1):154-8. doi:10.1016/J.VACCINE.2012.10.078

54. Bhandari N, Rongsen-Chandola T, Bavdekar A, et al. Efficacy of a monovalent human-bovine $(116 \mathrm{E})$ rotavirus vaccine in Indian infants: a randomised, double-blind, placebo-controlled trial. Lancet. 2014;383(9935):2136-43. doi:10.1016/S0140-6736(13)62630-6

55. Press release: India-made rotavirus vaccine achieves World Health Organization prequalification - PATH. [cited 23 May 2018]. http:// www.path.org/news/press-room/860/

56. Bines JE, Danchin M, Jackson P, et al. Safety and immunogenicity of RV3-BB human neonatal rotavirus vaccine administered at birth or in infancy: a randomised, double-blind, placebo-controlled trial. Lancet Infect Dis. 2015;15(12):1389-97. doi:10.1016/S1473-3099 (15)00227-3

57. Seheri M, Nemarude L, Peenze I, et al. Update of rotavirus strains circulating in Africa from 2007 through 2011. Pediatr Infect Dis J. 2014;33:S76-S84. doi:10.1097/INF.0000000000000053

58. Moussa A, Ben Hadj Fredj M, Fodha I, et al. Distribution of rotavirus VP7 and VP4 genotypes circulating in Tunisia from 2009 to 2014: emergence of the genotype G12. J Med Microbiol. 2016;65(9):102837. doi:10.1099/jmm.0.000305

59. Patton JT. Rotavirus diversity and evolution in the post-vaccine world. Discov Med. 2012;13(68):85-97. http://www.ncbi.nlm.nih.gov/ pubmed/22284787

60. Bányai K, László B, Duque J, et al. Systematic review of regional and temporal trends in global rotavirus strain diversity in the pre rotavirus vaccine era: insights for understanding the impact of rotavirus vaccination programs. Vaccine. 2012;30:A122-30.

61. Leshem E, Lopman B, Glass R, et al. Distribution of rotavirus strains and strain-specific effectiveness of the rotavirus vaccine after its introduction: a systematic review and meta-analysis. Lancet Infect Dis. 2014;14(9):847-56. doi:10.1016/S1473-3099 (14)70832-1

Received: 7-02-2018 Accepted: 21-11-2018 